Laurinda Fátima da F. P. Guimarães Bragança

\title{
Síntese e Caracterização de Nanocristais de Co-Fe suportados em Sílicas Mesoporosas para Síntese de Fischer-Tropsch
}

\section{Tese de Doutorado}

Tese apresentada como requisito parcial para obtenção do grau de Doutor pelo programa de Pós Graduação em Química do Departamento de Química da PUC-Rio.

Orientador: Prof. Maria Isabel Pais da Silva 
Laurinda Fátima da F. P. Guimarães Bragança

\section{Síntese e Caracterização de Nanocristais de Co-Fe suportados em Sílicas Mesoporosas para Síntese de Fischer-Tropsch}

Tese apresentada como requisito parcial para obtenção do grau de Doutor em Ciências pelo Programa de Pós-Graduação em Química do Departamento de Química do Centro Técnico Científico da PUC-Rio. Aprovada pela Comissão Examinadora abaixo assinada.

Prof. Maria Isabel Pais da Silva Orientadora Departamento de Química - PUC- Rio

Prof. Fábio Barboza Passos

UFF

Prof. Fernando Benedicto Mainier UFF

Prof. Cristiane Assumpção Henriques UERJ

Dr ${ }^{\text {a }}$ Paula Mendes Jardim Departamento de Engenharia de Materiais- PUC-Rio

Prof. José Eugenio Leal

Coordenador Setorial do Centro Técnico Científico - PUC-Rio 
Todos os direitos reservados. É proibida a reprodução total ou parcial do trabalho sem autorização da universidade, da autora e do orientador.

\section{Laurinda Fátima da F. P. Guimarães Bragança}

Graduou-se em Engenharia Química na Universidade Federal Fluminense em 1979. Defendeu sua Dissertação de Mestrado em Fenômenos de Transporte em 1985 sob o título de "Separação de misturas líquidas em escoamento pelicular por radiação térmica", pela COPPE - Universidade Federal do Rio de Janeiro, tendo apresentado o trabalho "Separação de misturas líquidas em escoamento pelicular em canais inclinados" nos anais do VIII Congresso Brasileiro de Engenharia Mecânica - COBEM - 1985.

Ficha Catalográfica

Bragança, Laurinda Fátima da F. P. Guimarães

"Síntese e caracterização de nanocristais de Co-Fe suportados em sílicas mesoporosas para síntese de Fischer Tropsch" / Laurinda Fátima da F. P. Guimarães Bragança ; orientador: Maria Isabel Pais da Silva. - 2010.

$201 \mathrm{f}$. : il. (color.) ; $30 \mathrm{~cm}$

Tese (Doutorado em Química)-Pontifícia Universidade Católica do Rio de Janeiro, Rio de Janeiro, 2010.

Inclui bibliografia

1. Química - Teses. 2. Sílicas mesoporosas. 3. HMS. 5. SBA-15. 6. Amostras bimetálicas de cobalto e ferro. 7. síntese de Fischer-Tropsch. I. Silva, Maria Isabel Pais da. II. Pontifícia Universidade Católica do Rio de Janeiro. Departamento de Química. III. Título. 
Para minha GRANDE família, Álvaro meu eterno amigo e incentivador, Fernanda, minha filha e meu estímulo e aos meus pais. 


\section{Agradecimentos}

A Deus por ter me concedido saúde e determinação ao longo desses anos para concretização deste trabalho.

Aos meus pais, por terem sempre me apoiado e ajudado a encontrar forças para enfrentar mais esse desafio.

A minha orientadora Maria Isabel Pais da Silva, pelo apoio, empenho, pela forma serena e segura que conduziu este trabalho.

Ao Ronaldo Pedro da Silva, Roberto R. Avillez, Carla M. da Silva, Henrique Meira da Silva, pela assistência nas análises.

Ao Instituto de Química da Universidade Federal da Bahia.

Ao Instituto de Catálisis y petroquímica, CSIC, na Espanha.

Ao estagiário da Pós-Graduação Bruno Damacena de Souza, pela ajuda nas análises, e por todo carinho e dedicação demonstrados ao longo de sua permanência no departamento.

A Universidade Federal Fluminense e ao Departamento de Engenharia Química e Petróleo pela concessão de tempo e estímulo para a realização desse trabalho.

A PUC-Rio pelos auxílios concedidos, e acolhimento durante o desenvolvimento deste trabalho.

Aos componentes da Comissão Examinadora. 


\section{Resumo}

Bragança, Laurinda Fátima F. P. G.; Silva, Maria Isabel Pais da. Síntese e Caracterização de Nanocristais de Co-Fe suportados em Sílicas Mesoporosas para a Síntese de Fischer-Tropsch. Rio de Janeiro, 2010. 201p. Tese de Doutorado - Departamento de Química. Pontifícia Universidade Católica do Rio de Janeiro.

Neste trabalho foram sintetizadas amostras bimetálicas de cobalto e ferro suportadas em sílicas mesoporosas do tipo SBA-15 e HMS. A preparação das mesmas ocorreu pelo método de impregnação incipiente do ponto úmido com soluções aquosas de nitratos de Co e Fe para obtenção de amostras com 25\% p/p total de metal. Para estudos de efeitos comparativos das diversas propriedades, amostras monometálicas de $\mathrm{Co}$ ou $\mathrm{Fe}$ foram também preparadas com o mesmo teor metálico. Para investigar o efeito de diferentes direcionadores de estrutura, duas séries da sílica mesoporosa HMS foram preparadas utilizando dois tipos de direcionadores: dodecilamina (DDA) e tetradecilamina (TDA). As amostras foram caracterizadas por medidas de fisissorção de $\mathrm{N}_{2}$, análise de difração de Raios-X (DRX), redução com temperatura programada (RTP), microscopia eletrônica de transmissão (TEM), quimissorção de $\mathrm{H}_{2}$ e espectroscopia fotoeletrônica de raios-X (XPS). A quantidade de metal incorporado foi estimada por espectroscopia de absorção atômica (EAA). Os suportes foram também caracterizados por análise diferencial termogravimétrica (ATG/ATD). As curvas ATG evidenciaram que os direcionadores de estrutura foram, em grande parte, removidos até $700 \mathrm{~K}$. As propriedades de textura mostraram que após a introdução metálica no suporte SBA-15, a área específica, o volume de poros e o diâmetro de poro decresceram em menor extensão para a amostra bimetálica em relação às monometálicas. As análises de DRX detectaram a formação das fases de $\mathrm{Co}_{3} \mathrm{O}_{4}$ e $\mathrm{CoFe}_{2} \mathrm{O}_{4}$ para todas as amostras bimetálicas; sendo que para a amostra de Co-Fe/HMS (TDA), a fase $\alpha-\mathrm{Fe}_{2} \mathrm{O}_{3}$ foi também indicada. A presença do suporte HMS (TDA) resultou na formação de fases cristalitas de maiores dimensões. Os valores das espessuras de parede para os suportes do tipo HMS foram maiores em relação aos encontrados na literatura, sugerindo a formação de materiais mais estáveis. Os perfis de RTP foram similares entre as amostras monometálicas e bimetálicas, entretanto, 
maiores temperaturas de redução do óxido de cobalto na amostra bimetálica foi indicada em relação às amostras monometálicas de cobalto, no suporte HMS (TDA). Uma interação metal-suporte mais forte foi evidenciada para a amostra Co-Fe/SBA-15. As medidas de quimissorção de $\mathrm{H}_{2}$ indicaram valores das dispersões metálicas maiores para as amostras bimetálicas em comparação as monometálicas de ferro e menores em relação às monometálicas de cobalto. A partir das micrografias obtidas por MET, foi observada uma menor formação (percentual) de aglomerados para a amostra de Co-Fe/SBA-15 em relação à de Co-Fe/HMS (DDA). O espectro Co2p resultante da análise de XPS, para a amostra bimetálica de Co-Fe/HMS (DDA) indicou a presença de $\mathrm{Co}_{3} \mathrm{O}_{4}$. O catalisador de Co-Fe/HMS (DDA) apresentou maiores seletividades para $\mathrm{C}_{5+} \mathrm{e}$ álcoois em relação ao catalisador Fe/HMS (DDA) na síntese de Fischer-Tropsch. O parâmetro de probabilidade de crescimento da cadeia $(\alpha)$ foi maior para o catalisador bimetálico Co-Fe/HMS (DDA) em comparação ao catalisador CoFe/SBA-15. Ambos os catalisadores bimetálicos exibiram uma seletividade (\%) maior para a fração de leves, $\mathrm{C}_{2}-\mathrm{C}_{4}$.

\section{Palavras-Chave}

Silicas mesoporosas; HMS; SBA-15; amostras bimetálicas de cobalto e ferro; síntese de Fischer-Tropsch. 


\section{Abstract}

Bragança, Laurinda Fátima F. P. G.; Silva, Maria Isabel Pais da (Advisor). Synthesis and Characterization of Co-Fe Nanocrystals supported on Mesoporous Silicas for the Fischer-Tropsch Synthesis. Rio de Janeiro, 2010. 201p. Doctorade Tesis - Departamento de Química. Pontifícia Universidade Católica do Rio de Janeiro.

In this work, cobalt and iron bimetallic samples supported on SBA-15 or HMS mesoporous sílicas were prepared by incipient wetness impregnation. Cobalt nitrate and iron salts were used to obtain samples containing $25 \mathrm{wt} \%$ total of metal content. Also, a series of supported monometallic cobalt or iron samples were synthesized, with the same metal loading, and compared to bimetallic ones. In order, to investigate the effect of different templates, a series of HMS sílicas have been prepared using two types of structure direction: dodecylamine (DDA) and tetradecylamine (TDA). The samples were cheracterized by $\mathrm{N}_{2}$-physisorption, $\mathrm{X}$-ray diffraction (XRD), temperature programmed reduction (TPR), transmission electron microscopy (TEM), $\mathrm{H}_{2}$ chemisorption and X-ray photoelectron spectroscopy (XPS) analysis. The amount of incorporated metal was estimated by atomic absorption spectroscopy (AAS). The supports were, also, characterized by thermogravimetric (TGA), as well as differential thermal (DTA) analyses. The TGA/DTA curves showed that the templates were mainly removed until $700 \mathrm{~K}$. The textural properties revealed that after metal introduction to SBA-15 support, specific area, pore volume and pore diameter decreased in a lesser extension for the bimetallic sample compared to monometallic ones. XRD measurements detected the formation of $\mathrm{Co}_{3} \mathrm{O}_{4}$ and $\mathrm{CoFe}_{2} \mathrm{O}_{4}$ phases for all bimetallic samples. For $\mathrm{Co}-\mathrm{Fe} / \mathrm{HMS}$ (TDA) sample, the $\alpha-\mathrm{Fe}_{2} \mathrm{O}_{3}$ phase formation and a higher $\mathrm{Co}_{3} \mathrm{O}_{4} / \mathrm{CoFe}_{2} \mathrm{O}_{4}$ crystallite sizes were also observed. The wall thickness valour for HMS supports was higher than previously reported values, suggesting more stable materials. The TPR profiles indicated similar behavior between bimetallic and monometallic samples. In case of cobalt oxide on bimetallic sample, higher reduction temperatures were indicated compared to monometallic ones for HMS (TDA) support. A higher interaction metal-support was showed for Co-Fe/SBA- 
15. According to hydrogen chemisorption, the bimetallic samples dispersions were higher than iron monometallic and lower than cobalt monometallic samples. From TEM microphotografs, it was observed a lesser fraction of agglomerates to Co-Fe/SBA-15 than Co-Fe/HMS (DDA) sample. XPS spectrum of Co2p region indicated the presence of $\mathrm{Co}_{3} \mathrm{O}_{4}$ for the bimetallic sample, Co-Fe/HMS (DDA). The Fe-Co/HMS (DDA) bimetallic catalyst showed higher $\mathrm{C}_{5+}$ and alcohols selectivities than Fe/HMS (DDA) on the Fischer-Tropsch reaction. Also, the bimetallic cobalt and iron based catalyst supported on HMS (DDA) recorded a higher value for the chain growth parameter $(\alpha)$ than Co-Fe/SBA-15. Both bimetallic catalysts exhibited highter selectivities (\%) for lighter fractions, $\mathrm{C}_{2}-\mathrm{C}_{4}$.

\section{Keywords}

Mesoporous silicas; HMS; SBA-15; Co-Fe bimetallic samples; FischerTropsch synthesis. 


\section{Sumário}

$\begin{array}{ll}\text { 1. Introdução } & 20\end{array}$

1.1 Panorama mundial para uso da tecnologia GTL 20

1.2 Objetivos 23

1.2.1 Objetivo Principal 23

$\begin{array}{lll}1.2 .2 & \text { Objetivos Específicos } & 23\end{array}$

2. Revisão da Literatura 25

2.1 Gás Natural 25

2.2 Rotas tecnológicas para o gás natural 26

2.3 Comercialização das tecnologias GTL 28

2.4 Tecnologia GTL 32

2.4.1 Geração de Gás de Síntese 33

2.4.2 Conversão do gás de síntese 34

2.4.3 Hidroprocessamento 35

2.5 Fischer-Tropsch - Desenvolvimento Histórico 35

2.5.1 Fischer-Tropsch - Mecanismo de Síntese 39

2.5.2 Distribuição de produtos - Modelo ASF 40

2.5.3 Catalisador de Cobalto 43

2.5.4 Catalisador de ferro 50

2.5.5 Catalisadores bimetálicos $\quad 56$

2.5.6 Reatores FT-GTL 59

2.6 Peneiras Moleculares 62

2.6.1 Introdução 62

2.6.2 Sílicas Mesoporosas: M41S - SBA-15 - HMS 69

3. Metodologia Experimental e Métodos 93

3.1 Preparação dos Sistemas Catalíticos 93

3.1.1 Síntese da sílica mesoporosa SBA-15 93

3.1.2 Síntese das sílicas mesoporosas HMS (DDA)

e HMS (TDA) 93 
3.1.3 Preparação dos catalisadores suportados em SBA-15

3.1.4 Preparação dos catalisadores suportados em

HMS (DDA) e HMS (TDA) 95

3.2 Técnicas de Caracterização 95

3.2.1 Composição Química 95

3.2.2 Análise Termogravimétrica 96

3.2.3 Propriedades Texturais 96

$\begin{array}{ll}\text { 3.2.4 Difração de raios }-X & 97\end{array}$

3.2.5 Redução com temperatura programada (RTP) 98

3.2.6 Quimissorção de hidrogênio 98

3.2.7 Microscopia Eletrônica de Transmissão (MET) 100

3.2.8 Espectroscopia fotoeletrônica de raios-X (XPS) 101

3.2.9 Testes catalíticos na síntese de Fischer-Tropsch 101

4. Resultados e Discussões 103

4.1 Determinação da Composição Química 103

4.2 Análise Termogravimétrica 103

4.2.1 Silica Mesoporosa Hexagonal, HMS (DDA) 103

4.2.2 Silica Mesoporosa Hexagonal, HMS (TDA) 104

4.2.3 Silica Mesoporosa, SBA-15 106

$\begin{array}{lll}4.3 & \text { Propriedades Texturais } & 107\end{array}$

4.3.1 HMS e amostras suportadas na silica mesoporosa

HMS 107

4.3.2 SBA-15 e amostras suportadas na silica mesoporosa SBA-15 113

$\begin{array}{lll}4.4 & \text { Difração de raios-X } & 116\end{array}$

4. 4.1 HMS e amostras suportadas na sílica mesoporosa HMS 116

4.4.2 SBA-15 e amostras suportadas na sílica mesoporosa SBA-15 122

4.5 Redução com Temperatura Programada (RTP) 127

4.5.1 Amostras suportadas na sílica mesoporosa HMS 127

4.5.2 Amostras suportadas na sílica mesoporosa SBA-15 134

4.6 Quimissorção de hidrogênio 139 
4.6.1 Amostras suportadas na sílica mesoporosa HMS 139

4.6.2 Amostras suportadas na silica mesoporosa SBA-15 142

4.7 Microscopia Eletrônica de Transmissão 145

4.7.1 Amostras suportadas na sílica mesoporosa

HMS (DDA) 145

4.7.2 Amostras suportadas na sílica mesoporosa SBA-15 149

4.8 Espectroscopia fotoeletrônica de raios-X (XPS) 155

4.8.1 Amostras suportadas na sílica mesoporosa

HMS (DDA) 155

4.9 Testes catalíticos na síntese de Fischer-Tropsch 160

4.9.1 Amostras suportadas na sílica mesoporosa

HMS (DDA) $\quad 160$

4.9.1.1 Co/HMS (DDA) 160

4.9.1.2 Fe/HMS (DDA) 164

4.9.1.3 Co-Fe/HMS (DDA) 168

4.9.2 Amostras suportadas na sílica mesoporosa SBA-15 173

4.9.2.1 Co/SBA-15 173

4.9.2.2 Fe/SBA-15 176

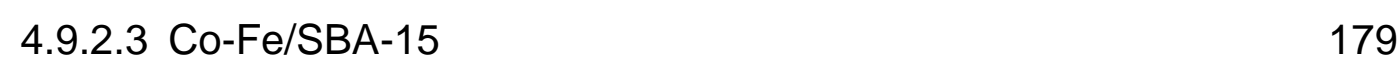

5. Conclusões e Sugestões 185

5.1 Conclusões 185

$\begin{array}{lll}5.2 & \text { Sugestões } & 190\end{array}$

$\begin{array}{ll}\text { 6. Referências Bibliográficas } & 191\end{array}$ 


\section{Lista de Tabelas}

Tabela 1 - Distribuição das reservas, produção e consumo de gás natural no mundo.

Tabela 2 - Características do diesel de refinaria e

Fischer-Tropsch.

Tabela 3 - Principais plantas GTL (em funcionamento

e previstas).

Tabela 4 - Dados termodinâmicos para reação

Fischer-Tropsch.

Tabela 5 - Hidrogenação de $\mathrm{CO}$ em presença de catalisadores de cobalto suportados em SCMM.

Tabela 6 - Área específica e volume de poros para catalisadores de ferro suportados.

Tabela 7 - Catalisadores típicos de plantas GTL.

Tabela 8 - Efeito do comprimento da cadeia do surfactante no tamanho do poro da MCM-41.

Tabela 9 - Propriedades texturais de sílicas mesoporosas e catalisadores de cobalto em diferentes suportes.

Tabela 10 - Composição química e propriedades texturais de Co/SBA-15 preparado com diversos precursores.

Tabela 11 - Resultados da síntese de FT para catalisadores de Co/SBA-15.

Tabela 12 - Comparação das propriedades texturais dos catalisadores CoS1 e CoS2.

Tabela 13 - Desempenho catalítico de CoS1 e CoS2 na síntese de FT.

Tabela 14 - Resultados dos testes catalíticos na síntese FT para catalisadores de Co/SBA-15 promovidos com Ru.

Tabela 15 - Resultados dos testes catalíticos para catalisadores de Co/SBA-15 com diferentes tamanhos de poro. 
Tabela 16 - Efeito da adição de Ru na atividade catalítica de catalisadores a base de cobalto suportados em MCM-41 e SBA-15.

Tabela 17 - Propriedades de textura e atividade em FT para Co/SBA-15 preparado com solução de etanol-Co(CH3COO)2 .

Tabela 18 - Conversão de CO, seletividade para C10-C20 e probabilidade de crescimento da cadeia para Co/SBA-15 e Fe/SBA-15, com diferentes diâmetros de poro.

Tabela 19 - Tamanho médio do cristalito de $\mathrm{Co} 3 \mathrm{O} 4$ para diferentes suportes.

Tabela 20 - Resultados dos testes catalíticos em FT para diferentes suportes.

Tabela 21 - Propriedades de textura e resultados DRX para os suportes HMS, Al-HMS, ZrO2/HMS e catalisadores de Co suportados.

Tabela 22 - Resultados da síntese de FT para catalisadores de Co/HMS.

Tabela 23 - Atividade catalítica e seletividade na síntese de Fischer-Tropsch para catalisadores de cobalto suportados em HMS.

Tabela 24 - Percentual metálico ( Co e/ou Fe ) determinado através de E.A.A.

Tabela 25 - Quantificação dos eventos de perda de massa das sílicas mesoporosas HMS e SBA-15.

Tabela 26 - Propriedades de textura para o suporte HMS e amostras suportadas.

Tabela 27 - Propriedades de textura para o suporte SBA-15 e amostras suportadas.

Tabela 28 - Resultados DRX para o suporte HMS

e amostras suportadas.

Tabela 29 - Resultados DRX para o suporte SBA-15

e amostras suportadas.

Tabela 30 - Consumo de hidrogênio e grau de redução das amostras monometálicas suportadas em HMS. 
Tabela 31 - Consumo de hidrogênio e grau de redução das amostras monometálicas suportadas em SBA-15.

Tabela 32 - Quantidade de hidrogênio adsorvida e área metálica das amostras de Co e/ou Fe suportadas em HMS.

Tabela 33 - Dispersão metálica e diâmetro de partícula, determinados por quimissorção de $\mathrm{H} 2$, para as amostras mono e bimetálicas suportadas em HMS.

Tabela 34 - Quantidade de hidrogênio adsorvida e área metálica das amostras de Co e/ou Fe suportadas em SBA-15.

Tabela 35 - Dispersão metálica e diâmetro de partícula, determinados por quimissorção de $\mathrm{H} 2$, para as amostras mono e bimetálicas suportadas em SBA-15.

Tabela 36 - Tamanho médio de partícula e aglomerado para amostras suportadas em HMS (DDA) e SBA-15.

Tabela 37 - Concentração atômica (\%) a partir de análise de XPS para amostras mono/bimetálica suportadas em HMS (DDA).

Tabela 38 - Energias de ligação $(\mathrm{eV})$ a partir de análise de XPS para amostras mono/bimetálica suportadas em HMS (DDA).

Tabela 39 - Conversão, Seletividade e probabilidade de crescimento da cadeia para o catalisador de Co/HMS (DDA).

Tabela 40 - Conversão, Seletividade e probabilidade de crescimento da cadeia para o catalisador de Fe/HMS (DDA).

Tabela 41 - Conversão, seletividade e probabilidade de crescimento da cadeia para o catalisador Co-Fe/HMS (DDA).

Tabela 42 - Conversão, seletividade e probabilidade de crescimento da cadeia para o catalisador Co/SBA-15.

Tabela 43 - Conversão, seletividade e probabilidade de crescimento da cadeia para o catalisador Fe/SBA-15.

Tabela 44 - Conversão, seletividade e probabilidade de crescimento da cadeia para o catalisador Co-Fe/SBA-15. 


\section{Lista de Figuras}

Figura 1 - Rotas GTL (Gas to Liquids).

Figura 2 - Valor dos produtos derivados de $1 \mathrm{MBTU}$ de Gás Natural.

Figura 3 - Estágios da tecnologia GTL.

Figura 4 - Esquemas de mecanismos para as reações de Fischer-Tropsch: carbeno (a), hidroxicarbeno (b) e inserção de $\mathrm{CO}$ (c).

Figura 5 - Seletividade de hidrocarbonetos baseada na equação ASF.

Figura 6 - Esquemas de Reatores Típicos para a

Síntese de FT.

Figura 7 - Estruturas mesoporosas do tipo M41S:

(a) hexagonal, (b) cúbica e (c) lamelar.

Figura 8 - Partícula de Si-MCM-41 - arranjo hexagonal.

Figura 9 - Mecanismos possíveis para formação de MCM-41.

Figura 10 - Difração de raios- $X$ da sílica mesoporosa , MCM-41, Calcinada.

Figura 11 - Imagens SEM e TEM de sílica mesoporosa SBA-15 preparada com TMOS como fonte de sílica.

Figura 12 - Imagens SEM da SBA-15 preparada com

TEOS, como fonte de sílica.

Figura 13 - Imagens MET da sílica mesoporosa, HMS.

Figura 14 - Curvas ATG/ATD da sílica mesoporosa hexagonal, HMS (DDA), não calcinada.

Figura 15 - Curvas ATG/ATD da sílica mesoporosa hexagonal, HMS (TDA), não calcinada.

Figura 16 - Curvas ATG/ATD da sílica mesoporosa SBA-15, não calcinada.

Figura 17 - Isotermas de adsorção/dessorção de N2 do 
suporte HMS (DDA) e amostras mono/bimetálica suportadas.

Figura 18 - Isotermas de adsorção/dessorção de N2

do suporte HMS (TDA) e amostras mono/bimetálica

suportadas em HMS (TDA).

109

Figura 19 - Curva de distribuição de diâmetro de poros

para a amostra Co-Fe/HMS (DDA).

112

Figura 20 - Curva de distribuição de diâmetro de poros

para a amostra Co-Fe/HMS (TDA).

Figura 21 - Isotermas de adsorção/dessorção de N2 do

suporte SBA-15 e amostras mono/bimetálica suportadas

em SBA-15.

114

Figura 22 - Curva de distribuição de diâmetro de poros

para a amostra Co-Fe/SBA-15.

Figura 23 - Análise de DRX do suporte HMS (DDA)

e amostras mono/bimetálica suportadas em HMS (DDA).

Figura 24 - Análise de DRX do suporte HMS (TDA)

e amostras mono/bimetálicasuportadas em HMS (TDA).

118

Figura 25 - Análise de DRX do suporte SBA-15

e amostras mono/bimetálica suportadas em SBA-15.

123

Figura 26 - Análise de RTP para as amostras mono/bimetálica suportadas em HMS (DDA).

Figura 27 - Análise de RTP para as amostras mono/bimetálica suportadas em HMS (TDA).

Figura 28 - Análise de RTP para as amostras mono/bimetálica suportadas em SBA-15.

Figura 29 - Imagens obtidas por MET para a amostra Co/HMS (DDA) (a) e (b); histograma de distribuição de tamanho de partícula (d) e análise por difração de elétrons (c) da região assinalada em (b).

Figura 30 - Imagem obtida por MET de alta resolução para a amostra Fe/HMS e respectivo histograma de distribuição de tamanho de partícula.

Figura 31 - Imagens obtidas por MET para a amostra Co-Fe/HMS (DDA) (a) e (c); histograma de distribuição de tamanho de partícula (d), análise por difração de eletrons 
(b) da região assinalada em (a).

Figura 32 - Imagens MET da amostra Co-Fe/HMS (DDA)

e respectivos espectros EDS.

Figura 33 - Imagens obtidas por MET para a amostra

Co/SBA-15 (a) e (b); histograma de distribuição de

tamanho de partícula (d) e análise por difração de elétrons

(c) da região assinalada em (b).

Figura 34 - Imagem obtida por MET para a amostra

Co/SBA-15.

Figura 35 - Imagem obtida por MET de alta resolução para

a amostra Fe/SBA-15 e respectivo histograma de

distribuição de tamanho de partícula.

152

Figura 36 - Imagem obtida por MET da amostra

Co-Fe/SBA-15 e respectivos espectros EDS das regiões

(1) e (2).

153

Figura 37 - Histograma de distribuição de tamanho de

partícula da amostra Co-Fe/SBA-15 e imagem de maior resolução para Co-Fe/SBA-15.

154

Figura 38 - Espectros em alta resolução do Fe2p para

amostra de Fe/HMS (DDA).

156

Figura 39 - Espectros em alta resolução do Co2p para

amostra de Co/HMS(DDA).

157

Figura 40 - Espectros em alta resolução do Co2p

da amostra bimetálica Co-Fe/HMS (DDA).

158

Figura 41 - Espectros em alta resolução do Fe2p

da amostra bimetálica Co-Fe/HMS (DDA).

158

Figura 42 - Distribuição de produtos para o catalisador

Co/HMS (DDA)

162

Figura 43 - Diagrama da probabilidade de crescimento da

cadeia para produtos obtidos com Co/HMS (DDA).

162

Figura 44 - Distribuição de produtos para o catalisador

Fe/HMS (DDA).

Figura 45 - Diagrama da probabilidade de crescimento da

cadeia para produtos obtidos com Fe/HMS (DDA)

Figura 46 - Distribuição de produtos para o catalisador 
Co-Fe/HMS (DDA).

Figura 47 - Diagrama da probabilidade de crescimento

da cadeia para produtos obtidos com Co-Fe/HMS (DDA).

170

Figura 48 - Seletividade para hidrocarbonetos dos

catalisadores de Co/HMS (DDA), Fe/HMS (DDA) e

Co-Fe/HMS (DDA).

170

Figura 49 - Distribuição de hidrocarbonetos para

o catalisador Co/SBA-15.

174

Figura 50 - Diagrama da probabilidade de crescimento

da cadeia para produtos obtidos com Co/SBA-15.

175

Figura 51 - Distribuição de produtos para o catalisador

Fe/SBA-15.

177

Figura 52 - Diagrama da probabilidade de crescimento da cadeia para produtos obtidos com Fe/SBA-15.

178

Figura 53 - Distribuição de produtos para o catalisador de Co-Fe/SBA-15.

180

Figura 54 - Diagrama da probabilidade de crescimento da cadeia para produtos obtidos com Co-Fe/SBA-15.

180

Figura 55 - Seletividade para hidrocarbonetos dos

catalisadores de Co/SBA-15, Fe/SBA-15 e Co-Fe/SBA-15.

181

Figura 56 - Seletividade (\%) para olefinas dos

catalisadores Co-Fe/HMS (DDA) e Co-Fe/SBA-15.

182

Figura 57 - Seletividade (\%) para diversas frações dos

catalisadores bimetálicos Co-Fe/HMS (DDA) e Co-Fe/SBA-15.

183 